\title{
Decanoic Acid Reverse Micelle-Based Coacervates for Microextraction of Silver in Natural Waters Prior to Flame Atomic Absorption Spectrometry Determination
}

\author{
Marzieh Shokouhifar, Seyed Mohammad Hosseini, Mohammad Reza Jamali* and \\ Reyhaneh Rahnama
}

\author{
Department of Chemistry, Payame Noor University, Tehran, Iran
}

\begin{abstract}
In the present work, a novel, simple, and efficient method for the determination of silver in natural water samples was developed. The method is based on the extraction of silver with coacervate made up of decanoic acid reverse micelles and the subsequent determination by flame atomic absorption spectrometry (FAAS). Dithizone was used as a chelating agent. To obtain the best extraction results, some experimental parameters (such as coacervate composition, $\mathrm{pH}$, concentration of chelating agent, and ionic strength) affecting the extraction efficiency were investigated and optimized. Under optimum conditions, the calibration curve was linear in the concentration range of 5-200 $\mu \mathrm{g} \mathrm{L}^{-1}$, with the correlation coefficient (r) equal to 0.995 . The limit of detection and the enrichment factor were $1.6 \mu \mathrm{g} \mathrm{L} \mathrm{L}^{-1}$ and 15 , respectively. The method was successfully applied to the analysis of silver in natural water samples.
\end{abstract}

Keywords: coacervate extraction, decanoic acid, silver determination, water sample

\section{Introduction}

The widespread use of silver compounds and silvercontaining procedures in industry, medicine, jewelry, cloud seeding, and in the disinfection of drinking water has resulted in an increasing silver content in environmental samples. ${ }^{1}$ However, despite the positive contributions of silver, primarily in medicine and technology, high concentrations of silver or long-time exposure to silver can cause serious health problems in living organisms. ${ }^{2}$ Silver is considered to be toxic to humans and the recommendations of the World Health Organization (WHO) permit maximum concentrations of $0.1 \mathrm{mg} \mathrm{L}^{-1}$ of silver ions in drinking water disinfection, but the United States Environmental Protection Agency (USEPA) recommends $0.05 \mathrm{mg} \mathrm{L}^{-1}$ as the maximum. ${ }^{3}$ Thus, the determination of low concentrations of silver ions is of increasing interest. ${ }^{4,5}$ Several atomic spectrometric techniques such as flame and electrothermal atomic absorption spectrometry (FAAS and ETAAS), ${ }^{6-9}$ inductively coupled plasma optical emission spectrometry (ICP OES), ${ }^{10}$ and inductively coupled plasma mass spectrometry (ICP-MS) $)^{11-14}$ have been proposed for the determination of silver in different environmental samples. Although the development of modern analytical

*e-mail: mr_jamali@ymail.com instruments provides a great enhancement in analysis, in many cases the available analytical instrumentation does not demonstrate enough sensitivity for the analysis of natural samples. ${ }^{15}$ Nevertheless, the detection of metal trace elements in aqueous samples is difficult due to various factors, particularly their low concentration and the matrix effects. ${ }^{16}$ Several methods have been reported for the separation and preconcentration of metal ions, such as coprecipitation, ${ }^{17,18}$ liquid-liquid extraction (LLE), ${ }^{19,20}$ solid-phase extraction (SPE), ${ }^{21-23}$ and cloud point extraction (CPE). ${ }^{24-26}$

Recently, coacervates made up of vesicles ${ }^{27,28}$ and reversed micelles of alkyl carboxylic acids ${ }^{29}$ have been reported, which permit the extraction of analytes in a wide polarity range. The word "coacervate" is derived from the Latin "co" (together) and "acerv" (a heap). In the present study, we investigate the suitability of amphiphilebased coacervative microextraction for the separation of silver ions from water samples prior to FAAS detection. Amphiphile-based coacervates are water immiscible liquids that separate from the bulk of molecular aggregate solutions (e.g., aqueous or reverse micelles or vesicles) by the action of a dehydrating agent, namely temperature, $\mathrm{pH}$, electrolyte or a non-solvent for the aggregates.30,31 After separation, the coacervate, a low volume phase, contains a high concentration of amphiphiles and therefore of 
binding sites. In consequence, high extraction efficiencies can be achieved using low coacervate volumes which results in high concentration factors. Detailed studies on coacervation process of various alkanoic acid reverse micelles in miscible binary mixtures of water and a variety of protic and aprotic solvents and their potential for the extraction have been reported by Ruiz et al. ${ }^{29}$ This new separation/preconcentration technique has been used for the extraction of various contaminants such as bisphenols in different matrices, ${ }^{31-34}$ ochratoxin $\mathrm{A},{ }^{35}$ carcinogenic polycyclic aromatic hydrocarbons ${ }^{36}$ prior to their determination by liquid chromatography, and arsenic ${ }^{37}$ by electrothermal atomic absorption spectrometry. To the best of our knowledge, there is no previous literature report on the use of supramolecular solvent made up of decanoic acid reversed micelles and FAAS technique for the preconcentration and determination of silver ions in real samples. Dithizone (DTZ) was used as a complexing agent and potential parameters affecting the analytical performance of the proposed method were studied and optimized in detail.

\section{Experimental}

Instrumentation

A PG-990 (PG instrument Ltd., Lutterworth, United Kingdom) atomic absorption spectrometer, equipped with deuterium background correction, silver hollow cathode lamp, and an air-acetylene flame was used for silver determination. All data was acquired with the equipment software according to peak height. The operating conditions were: wavelength $328.1 \mathrm{~nm}$, spectral resolution $0.4 \mathrm{~nm}$, applied lamp current $5.0 \mathrm{~mA}$, air flow rate $10.0 \mathrm{~L} \mathrm{~min}^{-1}$, and acetylene flow rate $1.2 \mathrm{~L} \mathrm{~min}^{-1}$. A Hettich centrifuge (Model Universal 320R, Tuttlingen, Germany) was used for centrifugation. The $\mathrm{pH}$ values were measured with a Metrohm pH-meter (model 827, Herisau, Switzerland) supplied with a glass-combined electrode.

\section{Reagents and solutions}

All reagents used were of analytical reagent grade. Deionized water was used throughout the experiments. A stock standard solution of silver $\left(1000.0 \mathrm{mg} \mathrm{L}^{-1}\right)$ was prepared by dissolving appropriate amounts of silver nitrate (Merck, Darmstadt, Germany) in deionized water containing $1.0 \mathrm{~mL}$ concentrated nitric acid (Merck, Darmstadt, Germany) in a $100 \mathrm{~mL}$ volumetric flask and diluted to mark with the deionized water and stored in the dark. Working solutions were prepared daily from the stock solutions by stepwise dilution. A $0.010 \mathrm{~mol} \mathrm{~L}^{-1}$ solution of dithizone (Merck, Darmstadt, Germany) was prepared in pure acetone. Decanoic acid was purchased from Fluka (Buchs, Switzerland). All glass vessels used for trace analysis were stored in $10 \%$ nitric acid for at least $24 \mathrm{~h}$ and washed four times with doubly distilled water before use.

\section{Extraction procedure}

For the coacervate extraction (CAE), aliquots of $15.0 \mathrm{~mL}$ of the sample or standard solution containing the silver ion (5-200 $\left.\mu \mathrm{g} \mathrm{L}^{-1}\right)$ was placed into the glass centrifuge tube. The $\mathrm{pH}$ of the solution was adjusted to $\mathrm{pH} 1.0$ via addition of $\mathrm{H}_{2} \mathrm{SO}_{4}$ and $0.15 \mathrm{~mL}$ of $1.0 \times 10^{-2} \mathrm{~mol} \mathrm{~L}^{-1}$ dithizone solution was added into the sample solution. Further, a binary solution containing $600 \mu \mathrm{L}$ of ethanol and $200 \mu \mathrm{L}$ of decanoic acid was injected into the sample solution using a syringe. Then, the mixture was gently shaken. After shaking, the solution became turbid and water immiscible coacervate made up of decanoic acid reverse micelles was formed. The extraction was accomplished in 1 min under shaking. Next, the mixture was centrifuged at $4000 \mathrm{rpm}$ for $5 \mathrm{~min}$ to accelerate the separation of the coacervate from the bulk solution. The aqueous phase was then separated completely by a syringe. Later, the coacervate phase was dissolved and made up to $1.0 \mathrm{~mL}$ by adding ethanol. The resultant solution was introduced into the flame by conventional aspiration.

\section{Results and Discussion}

The influence of the composition of the coacervate and different operational parameters (e.g., pH, ligand concentration, ionic strength, extraction time, etc.) on the recovery of silver was investigated. Triplicate extraction were performed for all experiments and the average of these results was reported in figures and tables.

\section{Effect of the coacervate composition}

Coacervation was only obtained in solvents that had the ability to dissolve alkyl carboxylic acids, permitted the self-assembly of these amphiphiles, and were water miscible. ${ }^{29}$ Therefore, in order to evaluate the influence of the coacervate composition on the extraction of silver, different composition of the binary solution of decanoic acid in tetrahydrofuran, ethanol, and acetone was investigated. The amount of decanoic acid and the type and amount of the solvent in the colloidal solution greatly influences both volume of extractant yielded and 
extraction efficiency. Water is only a minor component of the coacervate on account of its non-solvent characteristic for the decanoic acid reverse micelles. In order to select the solvent, a series of sample solutions were studied using $500 \mu \mathrm{L}$ of each solvent (tetrahydrofuran, ethanol, and acetone) containing $150 \mu \mathrm{L}$ of decanoic acid. The extraction recovery obtained for acetone, tetrahydrofuran, and ethanol were $95.2 \pm 1.5,95.5 \pm 1.3$ and $95.4 \pm 1.6 \%$, respectively. The results showed no statistically significant differences between the solvents. However, ethanol was selected as the solvent for subsequent experiments.

The effect of the amount of decanoic acid on the extraction recovery of silver was investigated. A series of solutions were made by dissolving different amounts of decanoic acid in $500 \mu \mathrm{L}$ of ethanol. As can be seen in Figure 1, the extraction efficiency enhanced with the increase in the amount of decanoic acid and reached a maximum in $150 \mu \mathrm{L}$ of decanoic acid and then remained constant. At lower amounts of decanoic acid, the extraction efficiency of silver was low probably due to the fact that low volumes of coacervate were unable to entrap quantitatively the hydrophobic Ag-DTZ complex. Therefore, $200 \mu \mathrm{L}$ of decanoic acid was used in further studies.

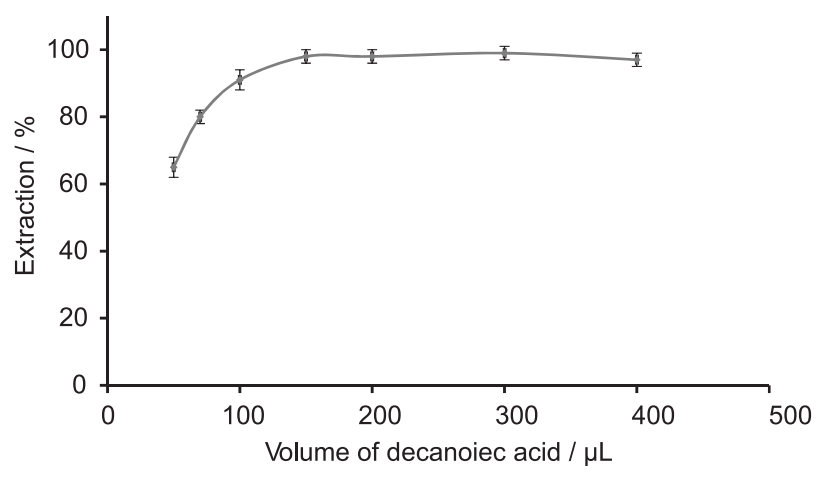

Figure 1. Effect of the amount of decanoic acid on the extraction of silver. Conditions: sample volume $15 \mathrm{~mL}, \mathrm{Ag}^{\mathrm{I}}$ concentration: $100.0 \mu \mathrm{g} \mathrm{L}{ }^{-1}, \mathrm{pH}$ : 1.0, dithizone concentration: $1.0 \times 10^{-4} \mathrm{~mol} \mathrm{~L}^{-1}$, volume of ethanol: $500 \mu \mathrm{L}$.

Also, the effect of the volume of ethanol on the coacervate phase formation and extraction recovery of silver was studied. The experimental conditions were fixed and included the use of different volumes of ethanol; 300 , $400,500,600,700,800,900$, and $1000 \mu \mathrm{L}$ containing $200 \mu \mathrm{L}$ of decanoic acid. In all mixtures, the coacervate phase was formed and the solution became turbid. As can be seen in Figure 2, maximal extraction efficiencies were obtained in the range of 500-800 $\mu \mathrm{L}$; beyond it, recoveries progressively decreased. Below this range, only a fraction of the decanoic acid was incorporated to the coacervate and, as a result, the recovery decreased. ${ }^{35}$ The decrease of extraction recovery at higher volumes of ethanol is probably due to the dissolution of a portion of the coacervate and AgDTZ complex in ethanol-water bulk solution. Therefore, $600 \mu \mathrm{L}$ of ethanol was selected as the optimum volume.

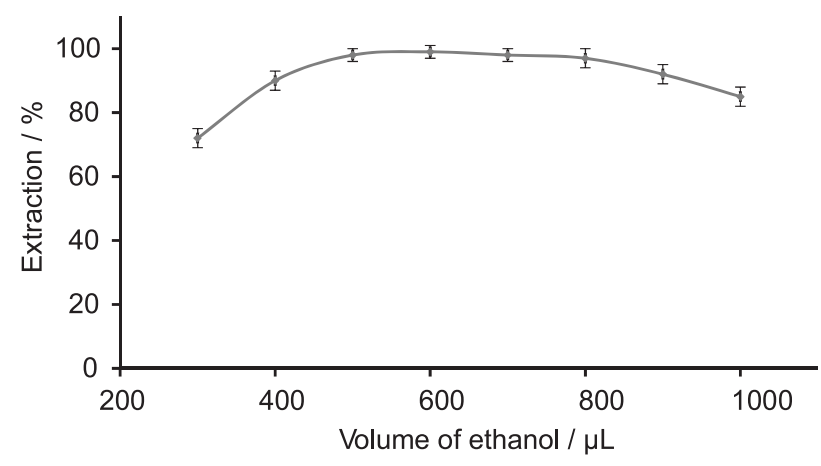

Figure 2. Effect of the volume of ethanol on the silver extraction. Conditions: sample volume $15 \mathrm{~mL}, \mathrm{Ag}^{\mathrm{I}}$ concentration: $100.0 \mu \mathrm{g} \mathrm{L}^{-1}$, $\mathrm{pH}$ : 1.0, dithizone concentration: $1.0 \times 10^{-4} \mathrm{~mol} \mathrm{~L}^{-1}$, volume of decanoic acid: $200 \mu \mathrm{L}$.

\section{Effect of $\mathrm{pH}$}

The $\mathrm{pH}$, which plays a unique role in metal-chelate formation and subsequent extraction, is proven to be a main parameter for CAE. The preliminary investigation showed that the silver ion is extracted by the coacervate phase effectively in acidic media. Sulfuric acid was chosen to adjust the $\mathrm{pH}$ of the experimental solutions because dithizone is stable in the presence of $\mathrm{H}_{2} \mathrm{SO}_{4}$. It should be noted that the presence of strong oxidizing acids such as $\mathrm{HNO}_{3}$ can decompose dithizone, and the presence of hydrochloric acid might cause the precipitation of silver. Coacervative extraction of silver was studied within the $\mathrm{pH}$ range of 0.5-4.0. The results show (Figure 3) that for $\mathrm{pH}$ below 1.5 , recovery of silver is close to $100 \%$. Hence, $\mathrm{pH}=1.0$ was chosen for the subsequent experiments. It should also be noted that a suitable condition for the selective extraction of a soft acid such as Ag by dithizone is attained in acidic conditions. ${ }^{38}$

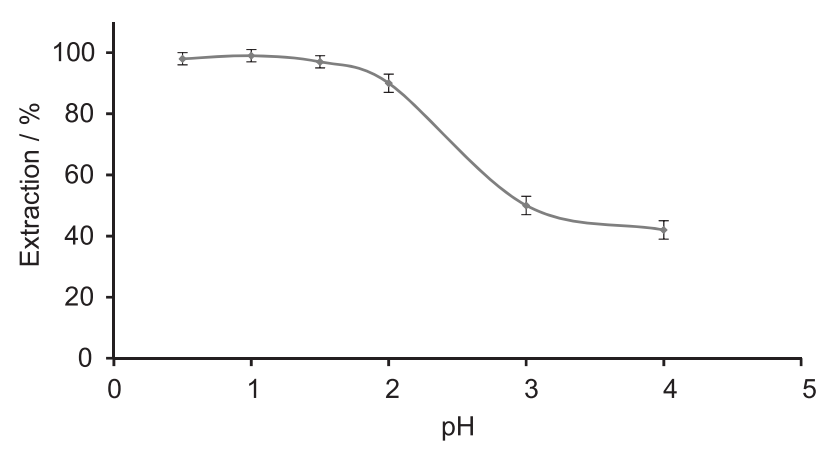

Figure 3. Effect of $\mathrm{pH}$ on the silver extraction. Conditions: sample volume $15 \mathrm{~mL}, \mathrm{Ag}^{\mathrm{I}}$ concentration: $100.0 \mu \mathrm{g} \mathrm{L}{ }^{-1}$, dithizone concentration: $1.0 \times 10^{-4} \mathrm{~mol} \mathrm{~L}^{-1}$, volume of decanoic acid: $200 \mu \mathrm{L}$, volume of ethanol: $600 \mu \mathrm{L}$. 


\section{Effect of dithizone concentration}

To accomplish the separation of metal ions by CAE, the use of a chelating agent is usually considered. In this case, a metal-chelate with sufficient hydrophobicity can be extracted to the small volume of coacervate phase. We selected and used dithizone due to its well-known reactivity with Ag. ${ }^{38}$ The effect of chelating reagent concentrations on the extraction recovery was investigated in the range of $1.0 \times 10^{-5}-3.0 \times 10^{-4} \mathrm{~mol} \mathrm{~L}^{-1}$. As shown in Figure 4, it was noticed that the extraction recovery increased with the increase of DTZ concentrations in the range of $1.0 \times 10^{-5}-3.0 \times 10^{-5} \mathrm{~mol} \mathrm{~L}^{-1}$, and at higher DTZ concentrations, the extraction recovery remained constant. Because of some other ions that could be present in real samples and probably react with DTZ, a concentration of $1.0 \times 10^{-4} \mathrm{~mol} \mathrm{~L}^{-1}$ was used in the experiments.

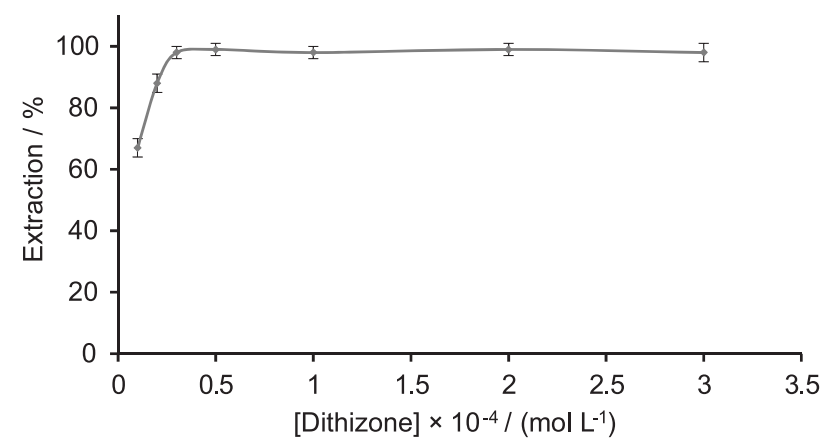

Figure 4. Effect of dithizone concentration on the silver extraction. Conditions: sample volume $15 \mathrm{~mL}, \mathrm{Ag}^{\mathrm{I}}$ concentration: $100.0 \mu \mathrm{g} \mathrm{L} \mathrm{L}^{-1}, \mathrm{pH}$ : 1.0 , volume of decanoic acid: $200 \mu \mathrm{L}$, volume of ethanol: $600 \mu \mathrm{L}$.

\section{Effect of centrifugation rate and time}

The effect of centrifugation rate on the extraction recovery of silver was studied in the range of 1000-6000 rpm. It was found that over $3000 \mathrm{rpm}$ the coacervate phase completely separated. Therefore, the rate of $4000 \mathrm{rpm}$ was selected as the optimum point. At the optimum rate, recovery of silver was investigated as a function of centrifugation time. Over $4 \mathrm{~min}$, extraction recovery was constant indicating a complete transfer of coacervate phase to the above of the solution and as such, 5 min was selected as the centrifugation time.

\section{Effect of ionic strength}

For investigating the influence of ionic strength on the performance of $\mathrm{CAE}$, various experiments were performed by adding different amounts of $\mathrm{NaNO}_{3}\left(0.0-2.0 \mathrm{~mol} \mathrm{~L}{ }^{-1}\right)$. Other experimental conditions were kept constant. The results showed that within experimental error limits, ionic strength had no appreciable effect upon extraction efficiency. Therefore, this method is a powerful sample preparation technique for saline solutions.

\section{Effect of foreign ions}

To assess the possible applications of the procedure, the effect of some possible interfering substances on the quantitative analysis of silver at $100.0 \mu \mathrm{g} \mathrm{\textrm {L } ^ { - 1 }}$ was tested. A substance was considered as interferent if it resulted in more than $5 \%$ variation in the extraction recovery. The results showed that, in excess of 10,000-fold of $\mathrm{Na}^{+}, \mathrm{K}^{+}$, $\mathrm{Ca}^{2+}, \mathrm{Mg}^{2+}, \mathrm{Ba}^{2+}, \mathrm{SO}_{4}{ }^{2-}, \mathrm{Cl}^{-}$and 1000 -fold of $\mathrm{Co}^{2+}, \mathrm{Mn}^{2+}$, $\mathrm{Ni}^{2+}, \mathrm{Cu}^{2+}, \mathrm{Hg}^{2+}, \mathrm{Zn}^{2+}, \mathrm{Cd}^{2+}, \mathrm{Pb}^{2+}, \mathrm{Fe}^{3+}, \mathrm{Fe}^{2+}, \mathrm{Mn}^{2+}, \mathrm{Al}^{3+}, \mathrm{Cd}^{2+}$, the ions had no significant interferences in the extraction and determination of silver. According to the results, the major ions in the real samples have no obvious influence on silver CAE under the selected conditions.

\section{Analytical figures of merit}

Under the optimum conditions, the CAE procedure was applied for the extraction and preconcentration of various standard solutions of silver and the calibration graph was linear within the range of 5-200 $\mu \mathrm{g} \mathrm{L}^{-1}$ with linear regression equation as Abs. $=0.0021 \mathrm{C}-0.0032$, $\mathrm{r}=0.995$, where Abs. is the absorbance intensity, $\mathrm{C}$ is the concentration of $\mathrm{Ag}$ in $\mu \mathrm{g} \mathrm{L^{-1 }}$ and $\mathrm{r}$ is the correlation coefficient. The limit of detection (LOD), defined as $3 \mathrm{~S}_{\mathrm{b}} / \mathrm{m}$ (where $\mathrm{S}_{\mathrm{b}}=0.0011$ and $\mathrm{m}=0.0021$ are the standard deviation of the blank and the slope of the calibration graph, respectively), was found to be $1.6 \mu \mathrm{g} \mathrm{L}^{-1}$. The enrichment factor was found to be equal to 15 . It was calculated as the ratio of silver concentration in the coacervate phase to the initial concentration in the aqueous phase. Also, the enhancement factor was 15.5. The enhancement factor was calculated as the ratio between the slopes of the calibration curves obtained with and without the preconcentration step. Repeatability was carried out by spiking blank samples at the concentration of $100.0 \mu \mathrm{g} \mathrm{L}^{-1}$ and the relative standard deviation (RSD) for ten replicate experiments was $2.4 \%$.

\section{Application to natural waters}

The proposed method was applied to the determination of silver from $15 \mathrm{~mL}$ of different water samples. Water samples, including tap water from our lab (Behshar, Iran), river water (Sari, Iran), mineral water (Damavand company, Iran), and sea water (Caspian Sea, Iran) were collected and the coacervate extraction method was applied to extract the 
Table 1. Determination of silver in natural water samples

\begin{tabular}{|c|c|c|c|}
\hline \multirow{2}{*}{ Sample } & \multicolumn{2}{|c|}{$\mathrm{Ag}^{\mathrm{I}}$ amount $/\left(\mu \mathrm{g} \mathrm{L}^{-1}\right)$} & \multirow{2}{*}{ Recovery / \% } \\
\hline & Added & Found $^{\mathrm{a}}$ & \\
\hline Tap water & 0.0 & n.d. & - \\
\hline \multirow[t]{2}{*}{ (Drinking water system of Behshahr, Iran) } & 50.0 & $49.6 \pm 0.5$ & 99.2 \\
\hline & 100.0 & $98.9 \pm 1.2$ & 98.9 \\
\hline Mineral water & 0.0 & n.d. & - \\
\hline \multirow[t]{2}{*}{ (Damavand mineral water, Iran) } & 50.0 & $49.2 \pm 0.6$ & 98.4 \\
\hline & 100.0 & $98.2 \pm 1.5$ & 98.2 \\
\hline River water & 0.0 & n.d. & - \\
\hline \multirow[t]{2}{*}{ (Tajan River, Sari, Iran) } & 50.0 & $49.1 \pm 0.6$ & 98.2 \\
\hline & 100.0 & $98.1 \pm 0.9$ & 98.1 \\
\hline Sea water & 0.0 & n.d. & - \\
\hline \multirow[t]{2}{*}{ (Caspian Sea water, Sari, Iran) } & 50.0 & $48.4 \pm 1.0$ & 96.8 \\
\hline & 100.0 & $97.9 \pm 2.1$ & 97.9 \\
\hline
\end{tabular}

${ }^{\mathrm{a}}$ Mean \pm standard deviation $(\mathrm{n}=3)$; n.d.: not detected.

silver. Each water sample was filtered in order to remove any suspended material. Recovery studies were also carried out after it was spiked to samples of known concentrations of silver at levels of 50 and $100 \mu \mathrm{g} \mathrm{L} \mathrm{L}^{-1}$. The obtained results are given in Table 1 . The recovery values calculated for the added standards ranged from 98.2 to $101.9 \%$, thus, confirming the accuracy of the procedure and its independence from the matrix effects. These results confirm the validity of the proposed preconcentration method.

\section{Comparing CAE with other methods}

A comparison of the presented method with other reported preconcentration methods for silver determination in water samples is given in Table 2. The presented method has low LOD, good enrichment factor and RSD and these characteristics are comparable or even better than most of the other methods in Table 2. All these results indicate that $\mathrm{CAE}$ is a reproducible, simple, and low cost technique that can be used for the preconcentration of metal ions like silver from water samples.

\section{Conclusion}

In the presented research, a simple, low cost, and environment-friendly CAE technique was developed. This technique is based on the extraction of Ag-DTZ chelate by a coacervate made up of decanoic acid reverse micelles prior to the determination by FAAS. The analyte can be selectively extracted from solutions at $\mathrm{pH} 1.0$ even in the presence of alkali, alkaline earth, transition, and heavy metal ions. The proposed method can be used successfully for the extraction and determination of silver from natural water samples.

Table 2. Comparison of CAE with other methods for determination of silver in water samples

\begin{tabular}{|c|c|c|c|c|c|}
\hline Method & $\mathrm{LOD}^{\mathrm{a}} /\left(\mu \mathrm{g} \mathrm{L}{ }^{-1}\right)$ & $\mathrm{RSD}^{\mathrm{b}} / \%$ & Sample volume / mL & $\mathrm{EF}^{\mathrm{c}}$ & Reference \\
\hline SPE-FAAS & 3.9 & 4.4 & 50 & 10 & 4 \\
\hline CPE-FAAS & 2.2 & 2.6 & 10 & 20 & 26 \\
\hline DLLME-FAAS & 1.2 & 1.5 & 8 & 16 & 39 \\
\hline CPE-FAAS & 10.0 & 1.8 & 14 & 35 & 40 \\
\hline SPE-FAAS & 1.0 & 2.9 & 100 & 18.7 & 41 \\
\hline SSE-FAAS & 1.9 & 3.6 & 10 & 18 & 42 \\
\hline DLLME-FAAS & 2.0 & 4.0 & 20 & 65 & 43 \\
\hline CAE-FAAS & 1.6 & 2.4 & 15 & 15 & present work \\
\hline
\end{tabular}

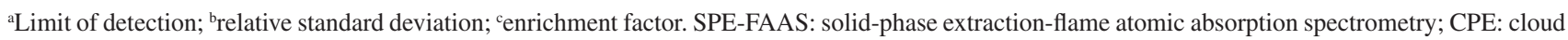
point extraction; DLLME: dispersive liquid-liquid microextraction; SSE: supramolecular solvent-based extraction; CAE: coacervate extraction. 


\section{Acknowledgment}

The authors thank the research council at the Payame Noor University for financial support.

\section{References}

1. World Health Organization (WHO); Silver and Silver Compounds: Environmental Aspects, Concise International Chemical Assessment Document, 44, WHO Press: Geneva, 2002.

2. Eisler, R.; Silver Hazards to Fish, Wildlife and Invertebrates: A Synoptic Review, US Department of the Interior, National Biological Service: Washington DC, 1996.

3. Pelkonen, K. H. O.; Heinonen-Tanski, H.; Hänninen, O. O. P.; Toxicology 2003, 186, 151.

4. Fathi, M. R.; Pourreza, N.; Purweis, S.; J. Chin. Chem. Soc. 2009, 56, 725 .

5. Mao, X.; Chen, H.; Liu, J.; Microchem. J. 1998, 59, 383.

6. Yavuz, E.; Tokalioglu, S.; Sahan, S.; J. Braz. Chem. Soc. 2013, 24, 736 .

7. Rastegarzadeh, S.; Pourreza, N.; Larki, A.; J. Ind. Eng. Chem. 2015, 24, 297.

8. Gerondi, F.; Arruda, M. A. Z.; Talanta 2012, 97, 395.

9. Lopez-Garcia, I.; Campillo, N.; Arnau-Jerez, I.; HernandezCordoba, M.; Spectrochim. Acta B 2003, 58, 1715.

10. Singh, R. P.; Pambid, E. R.; Analyst 1990, 115, 301.

11. Ndungu, K.; Ranville, M. A.; Franks, R. P.; Flegal, A. R.; Mar. Chem. 2006, 98, 109.

12. Yang, L.; Ralph, R. E.; J. Anal. At. Spectrom. 2002, 17, 88.

13. Hu, J.; Liu, Z.; Wang, H.; Anal. Chim. Acta 2002, 451, 329.

14. Chang, C. C.; Liu, H. T.; Jiang, S. J.; Anal. Chim. Acta 2003, 493, 213

15. Carasek, E.; Tonjes, J. W.; Scharf, M.; Talanta 2002, 56, 185.

16. Chena, J.; Xiao, S.; Wu, X.; Fang, K.; Liu, W.; Talanta 2005 , 67, 992.

17. Wang, Y.; Chen, M. L.; Wang, J. H.; J. Anal. Atom. Spectrom. 2006, 21, 535.

18. Soylak, M.; Tuzen, M.; J. Hazard. Mater. 2008, 152, 656.

19. Anthemidis, A. N.; Themelis, D. G.; Stratis, J. A.; Talanta 2001, $54,37$.

20. Pan, L.; Qin, Y. C.; Hu, B.; Jiang, Z. C.; Chem. Res. Chin. Univ. 2007, 23, 399.

21. Jamali, M. R.; Boromandi, A.; J. Braz. Chem. Soc. 2014, 25, 1078.

22. Dasbasi, T.; Sacmaci, S.; Sahan, S.; Kartal, S.; Ulgen, A.; Talanta 2013, 103, 1.
23. Karimi, M.; Shabani, A. M. H.; Dadfarnia, S.; J. Braz. Chem. Soc. 2016, 27, 144.

24. Rahnama, R.; Eram, S.; Jamali, M. R.; J. Braz. Chem. Soc. 2014, 25, 658.

25. Jalbani, N.; Soylak, M.; J. Ind. Eng. Chem. 2015, 29, 48.

26. Shemirani, F.; Rahnama Kozani, R.; Assadi, Y.; Microchim. Acta 2007, 157, 81.

27. Ruiz, F. J.; Rubio, S.; Perez-Bendito, D.; Anal. Chem. 2006, 78, 7229 .

28. Lopez-Jimenez, F. J.; Rubio, S.; Perez-Bendito, D.; J. Chromatogr. A 2008, 1195, 25.

29. Ruiz, F. J.; Rubio, S.; Perez-Bendito, D.; Anal. Chem. 2007, 79, 7473.

30. IUPAC; Compendium of Chemical Terminology, vol. 31, 1972, p. 611.

31. Gander, B.; Blanco-Prieto, M. J.; Thomasin, C.; Wandrey, C.; Hunkeler, D. In Encyclopedia of Pharmaceutical Technology; Swarbrick, J.; Boylan J. C., eds.; Marcel Dekker: New York, 2001, p. 481.

32. Ballesteros-Gomez, A.; Ruiz, S.; Rubio, F. J.; Perez-Bendito, D.; Anal. Chim. Acta 2007, 603, 51.

33. Garcia-Prieto, A.; Lunar, L.; Perez-Bendito, D.; Anal. Chim. Acta 2008, 617, 51.

34. Garcia-Prieto, A.; Lunar, L.; Rubio, S.; Perez-Bendito, D.; Anal. Chim. Acta 2008, 630, 19.

35. Garcia-Fonseca, S.; Ballesteros-Gomez, A.; Rubio, S.; PerezBendito, D.; Anal. Chim. Acta 2008, 617, 3.

36. Ballesteros-Gomez, A.; Rubio, S.; Perez-Bendito, D.; J. Chromatogr. A 2008, 1203, 168.

37. Amjadi, M.; Manzoori, J. L.; Taleb, Z.; Microchim. Acta 2010 , 169, 187.

38. Manzoori, J. L.; Karim-Nezhad, G.; Anal. Chim. Acta 2003, 484, 155.

39. Mohammadi, S. Z.; Afzali, D.; Taher, M. A.; Baghelani, Y. M.; Talanta 2009, 80, 875.

40. Tavallali, H.; Yazdandoust, S.; Yazdandoust, M.; Clean: Soil, Air, Water 2010, 38, 242.

41. Xiang, G.; Li, L.; Jiang, X.; He, L.; Fan, L.; J. Chil. Chem. Soc. 2013, 58, 2182.

42. Meng, L.; Cheng, J.; Yang, Y.; Water Sci. Technol. 2013, 69, 580.

43. Dasbasi, T.; Sacmaci, S.; Ulgen, A.; Kartal, S.; J. Ind. Eng. Chem. 2015, 28, 316.

Submitted: January 26, 2016

Published online: April 5, 2016 